Bundesgesundheitsbl 2013 · 56:1321-1328

DOI 10.1007/s00103-013-1787-1

Online publiziert: 29. August 2013

(c) Springer-Verlag Berlin Heidelberg 2013

A. Siedler · A. Grüber ${ }^{2}$ A. Mankertz ${ }^{3}$

${ }^{1}$ Abteilung für Infektionsepidemiologie, Fachgebiet Impfprävention, Robert Koch-Institut, Berlin

2 Deutsches Grünes Kreuz für Gesundheit e.V. (DGK), Marburg

${ }^{3}$ Nationales Referenzzentrum für Masern, Mumps, Röteln, Robert Koch-Institut, Berlin

\title{
Masern-Surveillance in Deutschland
}

\section{Vom Sentinel zur Meldepflicht}

\section{Hintergrund}

Im Herbst 1999 wurde die Arbeitsgemeinschaft Masern (AGM) als gemeinsame Initiative des Robert Koch-Instituts (RKI) mit mehreren Impfstoffherstellern und dem Deutschen Grünen Kreuz (DGK) gegründet. Im Zentrum der Aktivitäten stand ein Sentinel für die Masern-Surveillance, da es keine bundesweiten Meldedaten zu Masern gab. Vom DGK wurden bundesweit etwa 1200 pädiatrische und hausärztliche Arztpraxen rekrutiert, die freiwillig und unentgeltlich klinische Masern-Verdachtsfälle meldeten und $\mathrm{Pa}$ tientenproben zur Laboruntersuchung auf Masern entweder an regionale Laboratorien oder an das Nationale Referenzzentrum für Masern, Mumps und Röteln (NRZ MMR) sandten. Damit war das Sentinel das erste bundesweite Surveillancesystem für Masern in Deutschland.

Die Impfstoffhersteller finanzierten in der AGM die Feldarbeit und das Datenmanagement des DGK. Das RKI hatte die wissenschaftliche Federführung, erhielt jedoch zu keinem Zeitpunkt finanzielle Unterstützung von den Impfstoffherstellern. Im RKI wurden die Mehrzahl der Laboruntersuchungen sowie die statistischen Auswertungen durchgeführt. Ein Wissenschaftlicher Beirat beriet die AGM bei der Erhebung und Datenauswertung und diskutierte 2-mal jährlich auf einem Workshop mit dem DGK und dem RKI über die jeweils aktuellen Sentinel-Ergebnisse.

Das Hauptanliegen des Masern-Sentinels war es, Trends in der Masernepidemiologie zu beschreiben, Masernfälle zu charakterisieren und eine molekulare Surveillance der Masernvirus-Zirkulation vorzunehmen. Eine Inzidenzschätzung war dagegen nicht möglich, da die Sentinel-Praxen eine selbst selektierte Teilnehmergruppe mit einem jeweils unbekannten Einzugsbereich war.

Mit Inkrafttreten des Infektionsschutzgesetzes (IfSG) wurden die Masern im Jahr 2001 meldepflichtig [1]. Die Meldepflicht an das zuständige Gesundheitsamt besteht sowohl für den Arzt, der einen Patienten mit Masernverdacht behandelt ( $\$ 6.1$ IfSG) als auch für ein diagnostisches Labor, das eine Maserninfektion nachweist ( $\$ 7.1$ IfSG). Im Gesundheitsamt werden alle Informationen zum Patienten zusammengeführt, Kontaktdetails ermittelt und Infektionsschutzmaßnahmen getroffen. Anonymisierte fallbasierte Angaben zu allen gemeldeten Masernfällen werden vom Gesundheitsamt an die Landesstellen und von dort an das RKI übermittelt. Damit gab es ab dem Jahr 2001 zwei parallele Surveillancesysteme für Masern, die sich teilweise ergänzten. Nachdem über mehrere Jahre hinweg nur noch wenige Fälle im Sentinel gemeldet wurden, wurde es im April 2011 eingestellt. Die Ergebnisse aus den ersten Jahren des Masern-Sentinels wurden publiziert $[4,12,14,17]$.

Ziel der vorliegenden Arbeit ist es, die Erfahrungen mit der Sentinel-Surveillance von Masern zusammenzufassen, ihre Ergebnisse mit denen aus der Masern-Meldepflicht zu vergleichen und so die Stärken und Limitierungen des Sentinels im Zusammenhang mit der jeweiligen epidemiologischen Situation aufzuzeigen.

\section{Methoden}

\section{Sentinel-Surveillance}

Nach Informationen und Aufrufen durch das DGK in der Fachpresse meldeten sich pädiatrische und hausärztliche Arztpraxen freiwillig zur unentgeltlichen Mitarbeit im Sentinel an. Sie wurden monatlich vom DGK aufgefordert, über Patienten mit Masernverdacht in ihrer Praxis per Fragebogen zu berichten oder eine Nullmeldung abzugeben, wenn keine Patienten mit Masern behandelt wurden. Darüber hinaus wurden sie gebeten, bei Patienten mit Masernverdacht eine Maserndiagnostik in einem regionalen Labor oder im NRZ MMR am RKI zu veranlassen und die Befunde dem DGK mit einem ergänzenden Fragebogen zu übermitteln.

\section{Labormethoden am NRZ MMR}

Während regionale Laboratorien fast ausschließlich serologische Tests zum Nachweis von spezifischen Antikörpern durchführten, wurde im NRZ MMR darüber hinaus das Masernvirus (MV) mittels der Polymerasekettenreaktion (PCR) in Urin, Rachenabstrichen und seit 2003 in Zahntaschenflüssigkeit („oral fluid“) nachgewiesen. Die Untersuchungen waren für die Sentinel-Ärzte kostenfrei; vom NRZ wurden Entnahmekits für die Patientenproben zur Verfügung gestellt.

Die Tests auf masernvirusspezifische Antikörper (IgG und IgM) im Serum wurden im NRZ MMR mit dem Enzygnost Test der Firma Siemens vorgenommen 
Tab. 1 Teilnehmende Sentinel-Praxen nach Arztgruppen und Zahl der gemeldeten Masernfälle, stratifiziert nach Laboruntersuchung und -ergebnis sowie Alter und Impfstatus der Patienten und nach Komplikationen; Sentinel der Arbeitsgemeinschaft Masern (AGM), 2000-2010

\begin{tabular}{|c|c|c|c|c|c|c|c|c|c|c|c|c|}
\hline & \multicolumn{11}{|l|}{ Jahr } & \multirow{2}{*}{$\begin{array}{l}\text { Gesamt } \\
-(2000-2010)\end{array}$} \\
\hline & 2000 & 2001 & 2002 & 2003 & 2004 & 2005 & 2006 & 2007 & 2008 & 2009 & 2010 & \\
\hline \multicolumn{13}{|l|}{ Sentinel-Praxen der AGM } \\
\hline AGM-Praxen insgesamt (n) & 1254 & 1242 & 1256 & 1228 & 1210 & 1186 & 1130 & 1191 & 1146 & 1070 & 1069 & 1488 \\
\hline Rücksenderate (in \%) & 99 & 97 & 98 & 97 & 95 & 95 & 94 & 91 & 90 & 85 & 86 & 93 \\
\hline AGM-Pädiater (n) & 1006 & 998 & 1001 & 983 & 974 & 952 & 906 & 815 & 785 & 738 & 738 & 1034 \\
\hline Rücksenderate (in \%) & 100 & 99 & 99 & 98 & 97 & 96 & 95 & 93 & 92 & 88 & 88 & 95 \\
\hline AGM-Hausärzte (n) & 248 & 245 & 255 & 245 & 235 & 234 & 224 & 376 & 361 & 332 & 331 & 454 \\
\hline Rücksenderate (in \%) & 98 & 93 & 96 & 94 & 90 & 90 & 89 & 87 & 85 & 79 & 81 & 89 \\
\hline $\begin{array}{l}\text { AGM-Praxen mit mindestens } 1 \text { Masern- } \\
\text { verdachtsfall }(n)\end{array}$ & 255 & 250 & 164 & 74 & 34 & 30 & 76 & 31 & 23 & 22 & 18 & 554 \\
\hline Darunter: Pädiater (n) & 229 & 220 & 142 & 64 & 32 & 28 & 69 & 27 & 21 & 18 & 15 & 475 \\
\hline \multicolumn{13}{|l|}{ Masernfälle } \\
\hline Masernverdachtsfälle (n) & 903 & 989 & 1158 & 97 & 38 & 56 & 196 & 43 & 27 & 34 & 32 & 3573 \\
\hline Laboruntersucht (n) & 362 & 428 & 310 & 77 & 33 & 50 & 140 & 31 & 21 & 13 & 24 & 1489 \\
\hline Untersuchungsrate (\% von gemeldet) & 40 & 43 & 27 & 79 & 87 & 89 & 71 & 72 & 78 & 38 & 75 & 42 \\
\hline Laborbestätigt (n) & 214 & 254 & 182 & 18 & 3 & 11 & 84 & 12 & 6 & 5 & 12 & 801 \\
\hline Bestätigungsrate (\% von untersucht) & 59 & 59 & 59 & 23 & 9 & 22 & 60 & 39 & 29 & 38 & 50 & 54 \\
\hline Laborergebnis nicht eindeutig (n) & 45 & 63 & 43 & 8 & 3 & 5 & 16 & 7 & 13 & 5 & 7 & 215 \\
\hline Keine Masern laut Laborbefund (n) & 103 & 111 & 85 & 51 & 27 & 34 & 40 & 12 & 2 & 3 & 5 & 473 \\
\hline Ausschlussrate (\% von untersucht) & 28 & 26 & 27 & 66 & 82 & 68 & 29 & 39 & 10 & 23 & 21 & 32 \\
\hline Auswertbare Masernfälle gesamt (n) & 800 & 878 & 1073 & 46 & 11 & 22 & 156 & 31 & 25 & 31 & 27 & 3100 \\
\hline \multicolumn{13}{|l|}{ Masernfälle nach Alter } \\
\hline$<1 \mathrm{Jahr}$ & 55 & 43 & 49 & 8 & 5 & 2 & 29 & 3 & 7 & 4 & 0 & 205 \\
\hline 1 bis 4 Jahre & 337 & 358 & 443 & 21 & 5 & 8 & 57 & 8 & 8 & 12 & 10 & 1267 \\
\hline 5 bis 9 Jahre & 237 & 307 & 404 & 11 & 0 & 7 & 31 & 8 & 5 & 5 & 8 & 1023 \\
\hline 10 bis 14 Jahre & 128 & 127 & 146 & 5 & 1 & 2 & 25 & 10 & 1 & 8 & 7 & 460 \\
\hline 15 bis 19 Jahre & 28 & 30 & 20 & 1 & 0 & 1 & 10 & 1 & 2 & 2 & 0 & 95 \\
\hline 20 Jahre und älter & 15 & 13 & 11 & 0 & 0 & 2 & 4 & 1 & 2 & 0 & 2 & 50 \\
\hline \multicolumn{13}{|c|}{ Masernfälle nach Impfstatus und Gründen, nicht geimpft zu sein } \\
\hline Unbekannter Impfstatus & 34 & 32 & 42 & 0 & 1 & 2 & 21 & 1 & 2 & 1 & 3 & 139 \\
\hline \multicolumn{13}{|l|}{ Ungeimpft weil } \\
\hline - Unbekannte Gründe & 370 & 299 & 203 & 15 & 7 & 7 & 69 & 12 & 12 & 14 & 6 & 1014 \\
\hline - Impfung nicht gewünscht & 251 & 380 & 677 & 13 & 0 & 5 & 22 & 13 & 6 & 4 & 12 & 1383 \\
\hline - Impfung vergessen & 84 & 79 & 71 & 3 & 0 & 4 & 17 & 0 & 0 & 3 & 4 & 265 \\
\hline - Medizinische Kontraindikation & 16 & 24 & 6 & 0 & 0 & 1 & 3 & 0 & 0 & 0 & 0 & 50 \\
\hline 1-mal geimpft & 43 & 60 & 64 & 11 & 3 & 2 & 21 & 5 & 2 & 6 & 0 & 217 \\
\hline 2-mal geimpft & 2 & 4 & 10 & 4 & 0 & 1 & 3 & 0 & 3 & 3 & 2 & 32 \\
\hline Komplikationen der Masern ( $n$ ) & n.a. & 131 & 154 & 10 & 2 & 6 & 10 & 4 & 4 & 6 & 5 & 323 \\
\hline Anteil an gesamt (\%) & & 15 & 14 & 22 & 18 & 27 & 6 & 13 & 16 & 19 & 19 & 11 \\
\hline
\end{tabular}

$[12,17]$. Die Durchführung der PCR zum Nachweis und zur Typisierung des MVGenoms in Rachenabstrichen, Urin und „oral fluid“ wurde bereits beschrieben [5].

\section{Datenmanagement}

im Sentinel und für

Masernmeldungen nach IfSG

Dateneingabe und -speicherung der Sentinel-Daten erfolgten beim DGK. Die
Datensätze enthielten neben der Identifikationsnummer der Sentinel-Praxis alle Informationen aus den fallbasierten Fragebögen: Geburtsmonat und -jahr sowie Geschlecht des Patienten, Erkrankungsbeginn, Diagnosebestätigung, Komplikationen (seit 2001), Impfstatus, wahrscheinliche Infektionsquelle, Zugehörigkeit zu einem Ausbruch (jedoch keine Querverbindung zu anderen Fällen).
Vom DGK wurden monatlich alle Datensätze mit neuen Fällen, mit Änderungen in vorhandenen Datensätzen der Fälle und in den Adressdaten der Sentinel-Praxen sowie eine Übersicht über die Meldeaktivität jeder einzelnen SentinelPraxis an das RKI übermittelt. Damit wurden die deskriptiven Auswertungen zur Anzahl und Verteilung gemeldeter Masernfälle im Sentinel ständig aktualisiert. 
Die Daten am NRZ MMR wurden separat eingegeben und gespeichert. Ein Link zwischen beiden Datenbanken war mangels eines gemeinsamen Identifikators nicht möglich.

Über den Ablauf der Datenübermittlung und Speicherung der IfSG-Meldedaten im elektronischen Meldesystem SurvNet am RKI wurde an anderer Stelle berichtet [3]. Inzidenzen wurden mithilfe von RKI-SurvStat ermittelt und in Fällen pro 100.000 Einwohner pro Jahr angegeben [10].

\section{Deskriptive Analyse}

In die deskriptive Analyse wurden alle monatlichen Fallmeldungen von Januar 2000 bis Dezember 2010 aus der AGMDatenbank am RKI einbezogen. Die Rücksenderate war definiert als Anteil der Sentinel-Praxen mit tatsächlich erfolgter Monatsmeldung an der Zahl angemeldeter Praxen. Fallzahlen wurden stratifiziert nach Jahr der Erkrankung, Alter der Patienten, Impfstatus, Laborergebnis und nach Komplikationen. Die Zahl der Arztpraxen mit monatlicher Meldung bildete den Nenner für die Fallzahlen pro Monat. Die jährliche Verteilung der Masernfälle nach Alter und Region im Sentinel wurde den nach IfSG übermittelten Meldedaten zu Masernfällen aus den Jahren 2001 bis 2010 gegenübergestellt.

\section{Ergebnisse}

\section{Sentinel-Praxen}

Von Januar 2000 bis Dezember 2010 waren insgesamt 1488 Arztpraxen zur Teilnahme bei der AGM angemeldet und hatten mindestens eine Meldung abgegeben. Unter ihnen waren 1034 pädiatrische Praxen (etwa $15 \%$ aller niedergelassenen Kinderärzte in Deutschland) und 454 Allgemeinmediziner und hausärztlich tätige Internisten (etwa $1 \%$ aller Hausarztpraxen) (• Tab. 1).

Während die Zahl der pädiatrischen Praxen im Sentinel von 1006 im Jahr 2000 auf 738 im Jahr 2010 abnahm, stieg die Zahl der teilnehmenden Hausärzte zunächst von 248 auf 376 im Jahr 2007 und ging in den letzten beiden Jahren wieder auf 330 Teilnehmer zurück. Die Rücksenderate war in beiden Arztgruppen rück-

Bundesgesundheitsbl 2013 · 56:1321-1328

c) Springer-Verlag Berlin Heidelberg 2013

\section{A. Siedler · A. Grüber · A. Mankertz Masern-Surveillance in Deutschland. Vom Sentinel zur Meldepflicht}

\section{Zusammenfassung}

Am bundesweiten Sentinel der Arbeitsgemeinschaft Masern (AGM) nahmen von September 1999 bis März 2011 Arztpraxen freiwillig und unentgeltlich teil. Seit 2001 gab es mit der Masern-Meldepflicht in Deutschland 2 parallele Surveillancesysteme. Ziel der hier vorgestellten Analyse war die Bewertung der Stärken und Grenzen des Sentinels im Vergleich zur Meldepflicht. AGM-Praxen wurden monatlich aufgefordert, bei Patienten mit Masernverdacht fallbezogene Fragebögen oder Nullmeldungen abzugeben. Klinische Proben wurden zur Diagnoseprüfung an regionale Laboratorien zum Nachweis von IgM und IgG im Serum oder an das Nationale Referenzzentrum (NRZ) Masern, Mumps, Röteln eingesandt. Dort wurde das Masernvirus in Urin, Rachen- und Zahntaschenabstrichen nachgewiesen und genotypisiert. Von Januar 2000 bis Dezember 2010 übermittelten 554 von 1488 AGM-Praxen insgesamt 3573 Masern-Verdachtsfälle und 1489 Laborproben, 934 Praxen sahen keine Masern. Die Maserndiagnose wurde 801 -mal bestätigt, 473-mal

\section{Measles surveillance in Germany. From sentinel to mandatory surveillance}

\section{Abstract}

From September 1999 to March 2011, sentinel surveillance of measles was conducted by a self-selected sample of private physicians in Germany. From 2001, when mandatory surveillance for measles was established, two surveillance systems worked in parallel. The aim of this article is to summarize the strengths and limitations of sentinel versus mandatory surveillance. Active monthly reporting included case-based questionnaires on patients with (suspected) measles or zeroreporting. For confirmation of measles, the diagnostic patient specimens were sent to regional laboratories for serological tests or to the National Reference Laboratory (NRC). In the NRC in addition to serological tests measles-virus (MV) detection by PCR in urine, throat swabs, and oral fluid (since 2003) as well as MVgenotyping was offered. From January 2000 to December 2010, 934 out of 1,488 participating sentinel-practices did not see any measles case, while 554 reported 3,573 suspected cases. Measles was confirmed by laboratory testing in 801 cases, excluded in 473 cases, and the diagnosis remained uncertain in 215 cases. Of 3,100 an- ausgeschlossen, 215-mal war der Laborbefund nicht eindeutig. Die Mehrzahl der Fälle war ungeimpft (87\%), überwiegend wegen Ablehnung der Masernimpfung ( $n=1383)$. Die Bestätigungsrate war bei Geimpften niedriger als bei Ungeimpften (19\% vs. 63\%). Ab 2006 unterschieden sich die im Sentinel erfassten Masernfälle in ihrer Verteilung nach Alter und Region stark von den Fällen aus der Meldepflicht. Der Anteil der Laborproben aus AGM-Praxen an den NRZ-Einsendungen ging von $>50 \%$ (bis 2002) auf $<5 \%$ (ab 2007) zurück. Das Sentinel beschrieb zuverlässig Trends und die Verteilung von Masern in Deutschland, ergänzte präventionsrelevante Angaben und spielte eine entscheidende Rolle für die Labordiagnostik. Da die Meldepflicht etabliert war und das Sentinel nicht mehr die epidemiologische Situation reflektierte, wurde es im April 2011 eingestellt.

Schlüsselwörter

Masern · Surveillance $\cdot$ Sentinel $\cdot$ Meldepflicht alyzed cases, $2,712(87 \%)$ were unvaccinated, $217(7 \%)$ and 32 (1\%) were vaccinated with one or two doses, respectively, and for $139(4 \%)$ cases the vaccination status was unknown. The main reason for not being vaccinated against measles was refusal $(n=1,383)$. The confirmation rate was lower in the vaccinated than in the unvaccinated patients (19\% vs. $63 \%$ ). Since 2006 , sentinel-cases have differed from notified cases by region and age. The proportion of sentinel cases from all NRCinvestigated cases decreased from more than $50 \%$ (2002) to less than 5\% (since 2007). Sentinel surveillance allowed for the detection of trends, delivered additional information for measles prevention, and played a major role in measles diagnostics. Since mandatory surveillance was established and sentinel surveillance no longer reflected the epidemiologic situation, the latter was abandoned in April 2011.

\section{Keywords}

Measles · Surveillance - Sentinel system . Mandatory surveillance 
Tab. 2 Gemeldete Masernverdachtsfälle nach Impfstatus und Laboruntersuchung, Sentinel der Arbeitsgemeinschaft Masern (AGM)

\begin{tabular}{|c|c|c|c|c|c|}
\hline Impfstatus & 1-mal geimpft & 2-mal geimpft & Ungeimpft & Impfstatus unbekannt & Gesamt \\
\hline Gemeldete Fälle mit Masernverdacht insgesamt & 368 & 98 & 2936 & 171 & 3573 \\
\hline Nicht im Labor untersucht & 104 & 11 & 1901 & 68 & 2084 \\
\hline Fälle mit Masernverdacht und Laboruntersuchung & 264 & 87 & 1035 & 103 & 1489 \\
\hline Untersuchungsrate (Anteil an gemeldeten Verdachtsfällen) (\%) & 72 & 89 & 35 & 60 & 42 \\
\hline Laborbestätigte Masernfälle & 77 & 8 & 663 & 53 & 801 \\
\hline Bestätigungsrate (Anteil an laboruntersuchten Verdachtsfällen) (\%) & 29 & 9 & 64 & 51 & 54 \\
\hline Ausgeschlossene Fälle (laut Laborbefund keine Masern) & 151 & 66 & 224 & 32 & 473 \\
\hline Anteil an laboruntersuchten Verdachtsfällen (\%) & 57 & 76 & 22 & 31 & 32 \\
\hline Laborergebnis nicht eindeutig & 36 & 13 & 148 & 18 & 215 \\
\hline Anteil an laboruntersuchten Verdachtsfällen (\%) & 14 & 15 & 14 & 17 & 14 \\
\hline Auswertbare Masernfälle insgesamt & 217 & 32 & 2712 & 139 & 3100 \\
\hline$\%$ von gesamt & 7 & 1 & 87 & 4 & 100 \\
\hline
\end{tabular}

läufig von fast $100 \%$ zu Beginn der Sentinel-Erhebung auf $88 \%$ bei den Pädiatern und $81 \%$ bei den Hausärzten im Jahr 2010.

\section{Masernfälle und Laborergebnisse}

Von Januar 2000 bis Dezember 2010 wurden insgesamt 3573 Masernverdachtsfälle aus 554 Sentinel-Praxen gemeldet, 934 Praxen sahen keine Masernfälle, sondern gaben ausschließlich Nullmeldungen ab (• Tab. 1). Die meisten Praxen, die Masernfälle berichteten (92\%), hatten weniger als 10 Fälle pro Jahr. Die Mehrheit der Fälle (96\%) wurde von Pädiatern gemeldet (Daten nicht gezeigt).

Für 1489 Masernverdachtsfälle (42\%) wurde eine Laboruntersuchung durchgeführt, die in 801 Fällen (54\% der untersuchten) den klinischen Masernverdacht bestätigte. In 473 Fällen (32\% der untersuchten) konnten die Masern anhand des Laborbefundes ausgeschlossen werden. In den restlichen Fällen ( $n=215)$ gab es im Labor $z$. B. wegen nicht ausreichendem oder nicht zeitgerecht abgenommenem Material keinen eindeutigen Befund ( $\bullet$ Tab. 1$)$.

Stratifiziert nach Impfstatus wurde für 351 geimpfte Patienten mit Masernverdacht (75\% aller gemeldeten geimpften $\mathrm{Pa}$ tienten) im Vergleich zu 1035 ungeimpften Patienten (35\% der gemeldeten ungeimpften) sowie zu 103 Patienten mit unbekanntem Impfstatus (60\% dieser Gruppe, - Tab. 2) eine Laboruntersuchung veranlasst. Die Bestätigungsrate (Anteil der bestätigten Masern an allen im Labor untersuchten Fällen) betrug $29 \%$ bzw. $9 \%$ bei Patienten mit einer bzw. 2 Impfungen gegen- über $63 \%$ bei Ungeimpften und $57 \%$ bei Patienten mit unbekanntem Impfstatus.

Alle durch Laborbefund ausgeschlossenen Fälle $(n=473)$ wurden in der AGMDatenbank als gelöscht markiert, und es verblieben insgesamt 3100 Masernfälle zur weiteren Analyse.

\section{Epidemiologische Beschreibung der Masernfälle im Sentinel}

Von 3100 Masernpatienten waren 2712 (87\%) ungeimpft, 217 bzw. 32 (7 bzw. 1\%) waren 1-mal bzw. 2-mal geimpft, und für 139 (4\%) Patienten war der Impfstatus nicht bekannt (• Tab. 2). Von 176 geimpften Patienten war das Impfdatum bekannt. Von ihnen hatten 50 die letzte Masernimpfung weniger als 20 Tage vor Erkrankungsbeginn erhalten (Daten nicht gezeigt). Daher können maximal 199 der insgesamt 249 geimpften Fälle als Impfversager eingestuft werden.

Zum Grund der unterbliebenen Impfung hatten 1698 ungeimpfte Patienten Angaben gemacht: 1383 (81\%) Patienten hatten keine Masern-Impfung gewünscht, 265 hatten die Impfung vergessen, und bei 50 Patienten lag eine medizinische Kontraindikation vor.

Stratifiziert nach Alter fiel die Mehrheit der Masernfälle $(n=1267)$ in die Altersgruppe 1 bis 4 Jahre, gefolgt von den 5- bis-9-Jährigen ( $\mathrm{n}=1023), 460$ Kindern im Alter von 10 bis 14 Jahren und 205 Fällen im ersten Lebensjahr. Zur Altersgruppe 15 bis 19 Jahre zählten 95 Fälle, und 50 waren 20 Jahre oder älter.

Unter den seit dem Jahr 2001 berichteten 2309 Fällen wurden 335 (15\%) mit
Komplikationen der Masernerkrankung beobachtet. Als häufigste Komplikationen (Mehrfachnennung möglich) wurden Otitis media ( $\mathrm{n}=187)$ und Pneumonien $(n=115)$ genannt. Zentralnervöse Komplikationen traten 8-mal auf, und in 65 Fällen wurden sonstige nicht näher spezifizierte Komplikationen gemeldet (- Tab. 1). Die Komplikationshäufigkeit unterschied sich nicht zwischen geimpften und ungeimpften Fällen.

Die meisten Patienten hatten die Masern-Infektion in Deutschland erworben. Bei 2433 Fällen war die Infektionsquelle bekannt: $n=1086$ (45\%) hatten sich in der Schule oder im Kindergarten angesteckt, n=649 (27\%) in der Familie, n=311 (13\%) bei Freunden oder Verwandten, $\mathrm{n}=147$ (6\%) bei Freizeitaktivitäten und 236 bei anderen, nicht näher bezeichneten Gelegenheiten. Die meisten Masernfälle $(n=2435 ; 77 \%)$ gehörten zu MasernAusbrüchen (Daten nicht in Tabellen oder Abbildungen gezeigt).

\section{Masernfälle im AGM-Sentinel und in der Meldepflicht nach IfSG (2001-2010)}

Die Zahl der im Sentinel erfassten Masernfälle war in den Jahren 2001 und 2002 mit jeweils mehr als 800 Fällen und 0,71 bzw. 0,85 Fällen pro Sentinel-Praxis am höchsten (- Abb. 1a). Mit Ausnahme des Jahres 2006 ( $n=156$ oder 0,14 Fälle pro Sentinel-Praxis) lag in den Jahren 2003 bis 2010 die Zahl der pro Praxis gemeldeten Fälle unter 0,04 (• Abb. 1a).

Die Zahl der pro Sentinel-Praxis gemeldeten Fälle zeigte keine gute Überein- 
Inzidenzen (IfSG) und Fälle pro Praxis (AGM)

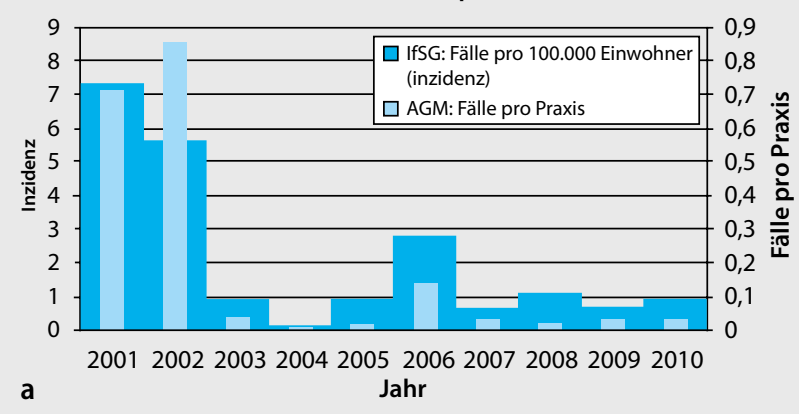

a

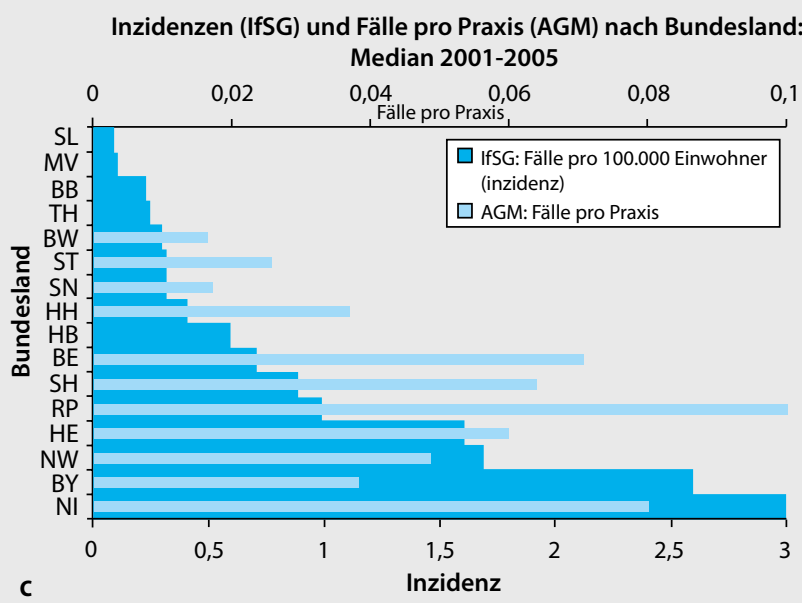

Altersverteilung der Masernfälle nach IfSG und AGM

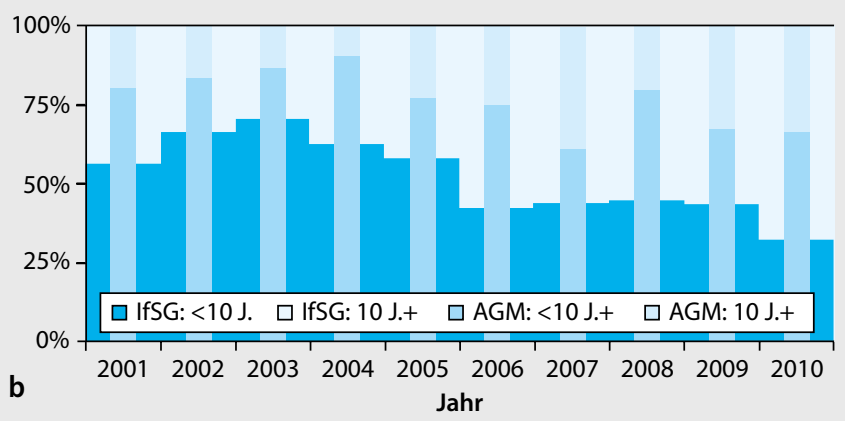

Inzidenzen (IfSG) und Fälle pro Praxis (AGM) nach Bundesland: Median 2006-2010
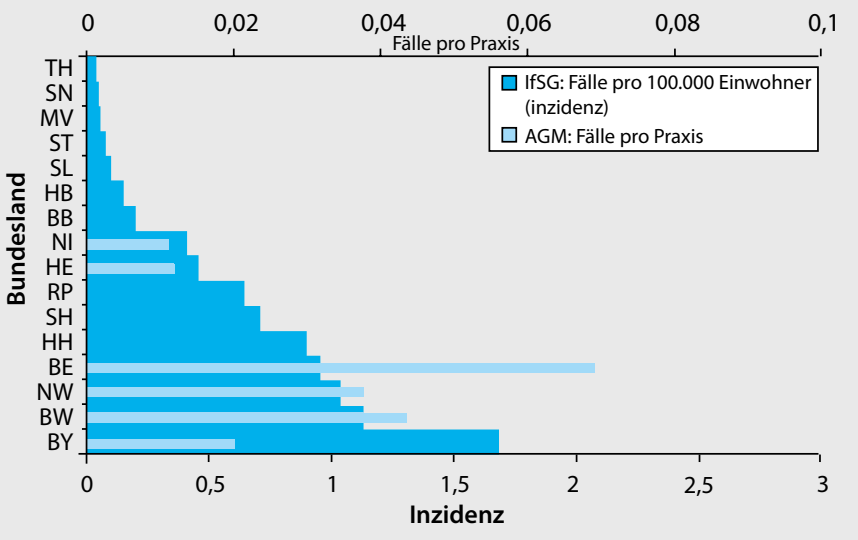

Abb. $1 \Delta$ Vergleich der Masernfälle, die nach Infektionsschutzgesetz (IfSG) übermittelt und die im Sentinel der Arbeitsgemeinschaft Masern (AGM) gemeldet wurden, nach a Fällen pro 100.000 Einwohner (Inzidenz; IfSG) bzw. Fällen pro Praxis (AGM), b Altersverteilung und c regionaler Verteilung nach Bundesländern, 2001 bis 2010. (BW Baden-Württemberg, $B Y$ Bayern, BE Berlin, $B B$ Brandenburg, HB Bremen, $H H$ Hamburg, HE Hessen, MV Mecklenburg-Vorpommern, NI Niedersachsen, NW Nordrhein-Westfalen, RP Rheinland-Pfalz, SL Saarland, SN Sachsen, ST Sachsen-Anhalt, SH Schleswig-Holstein, THThüringen)

stimmung mit den nach IfSG berechneten Masern-Inzidenzen. Obwohl beide Datenquellen in den Jahren 2001 und 2002 im Vergleich zu den folgenden Jahren höhere Werte zeigten, lag der höchste Wert nach IfSG im Jahr 2001 und nach AGM-Sentinel im Jahr 2002. Seit dem Jahr 2003 veränderte sich die Zahl der Masernfälle pro Sentinel-Praxis kaum und bildete die auftretenden Schwankungen in der Inzidenz der nach IfSG erfassten Fälle nicht ab. Einzige Ausnahme war das Jahr 2006, das durch einen großen Masernausbruch in Nordrhein-Westfalen geprägt war, der in beiden Systemen übereinstimmend zu höheren Werten führte (• Abb. 1a).

Während in den IfSG-Daten seit 2006 die Mehrheit der Masernfälle bei Personen ab einem Alter von 10 Jahren auftrat, lag im AGM-Sentinel der Anteil der Masernpatienten in diesem Alter im selben Zeitraum zwischen 9\% und 39\% (• Abb. 1b).

Ein Vergleich der Masern-Inzidenzen nach Bundesländern mit den Masern-
Fallzahlen pro Sentinel-Praxis und Bundesland zeigt im Median der Jahre 2001 bis 2005, dass die höchsten Werte in beiden Systemen in jeweils anderen Bundesländern beobachtet wurden (• Abb. 1c). Im Median der Jahre 2006 bis 2010 waren in beiden Erfassungssystemen zwar insgesamt niedrigere Werte in den Bundesländern zu verzeichnen, jedoch lag im AGMSentinel der Median der Fälle pro Sentinel-Praxis in 10 von 16 Bundesländern bei 0 . Nach IfSG lagen dagegen Fallmeldungen aus allen Bundesländern vor, und der Median der Inzidenz lag zwischen 0,04 (Thüringen) und 1,7 (Bayern).

\section{Laborergebnisse für das AGM-Sentinel im NRZ MMR}

An das NRZ MMR wurden von 293 AGMPraxen Proben von insgesamt $1238 \mathrm{~Pa}-$ tienten mit klinischem Masernverdacht eingesandt ( $\bullet$ Tab. 3). Durch den Laborbefund wurde der klinische Masernver- dacht in 617 Fällen bestätigt, 474-mal wurde eine Masern-Erkrankung ausgeschlossen, in 147 Fällen brachte der Laborbefund kein eindeutiges Ergebnis.

Die Altersverteilung der im NRZ MMR untersuchten Masernfälle unterschied sich nicht von der Altersverteilung der AGM-Fälle insgesamt. Der Anteil der AGM-Fälle an allen im NRZ MMR untersuchten Fällen zur Maserndiagnostik ging über den Beobachtungszeitraum stark zurück: Kamen in den Jahren 2000 bis 2002 mehr als $80 \%$ aller Einsendungen zur Maserndiagnostik an das NRZ MMR von Sentinel-Praxen der AGM, so lag dieser Anteil in den Jahren 2003 bis 2006 bei $28 \%$ und zwischen 2007 und 2010 nur noch bei $3 \%$.

Für insgesamt 480 Masern-Fälle aus dem AGM-Sentinel gelang eine Genotypisierung des Erregers. Während im Jahr 2000 der MV-Genotyp C2 noch am häufigsten gefunden wurde (in 47 von 109 sequenzierten Proben), daneben aber auch 
Tab.3 Fällezur Sicherung der klinischen Diagnose „Masern“ und zur Masernvirus (MV)-Genotypisierung im Nationalen Referenzzentrum für Masern, Mumps und Röteln (NRZMMR) insgesamt und darunter Einsendungen der Sentinel-Praxen aus der Arbeitsgemeinschaft Masern (AGM), 2000 bis 2010

\begin{tabular}{|c|c|c|c|c|c|c|c|c|c|c|c|c|}
\hline Jahr & 2000 & 2001 & 2002 & 2003 & 2004 & 2005 & 2006 & 2007 & 2008 & 2009 & 2010 & Gesamt \\
\hline $\begin{array}{l}\text { Gesamtzahl von Fällen zur Masern- } \\
\text { diagnostik am NRZ MMR }\end{array}$ & 331 & 373 & 382 & 257 & 60 & 161 & 482 & 287 & 584 & 325 & 351 & 3593 \\
\hline - Masern bestätigt & 191 & 218 & 223 & 126 & 6 & 68 & 253 & 108 & 233 & 121 & 198 & 1745 \\
\hline - Masern ausgeschlossen & 108 & 113 & 108 & 111 & 44 & 85 & 176 & 126 & 111 & 42 & 57 & 1081 \\
\hline - Ergebnis nicht eindeutig & 32 & 42 & 51 & 20 & 10 & 8 & 53 & 53 & 240 & 162 & 96 & 767 \\
\hline $\begin{array}{l}\text { Zahl der AGM-Fälle zur Maserndiagnostik } \\
\text { am NRZ MMR }\end{array}$ & 304 & 340 & 266 & 65 & 32 & 48 & 126 & 19 & 15 & 14 & 9 & 1238 \\
\hline - Masern bestätigt & 172 & 195 & 148 & 9 & 0 & 11 & 67 & 4 & 5 & 5 & 1 & 617 \\
\hline - Masern ausgeschlossen & 103 & 105 & 86 & 51 & 28 & 35 & 43 & 10 & 3 & 5 & 5 & 474 \\
\hline - Ergebnis nicht eindeutig & 29 & 40 & 32 & 5 & 4 & 2 & 16 & 5 & 7 & 4 & 3 & 147 \\
\hline Gesamtzahl Fälle mit MV-Genotypisierung & 121 & 174 & 187 & 113 & 4 & 48 & 220 & 81 & 189 & 151 & 181 & 1469 \\
\hline Häufigste MV-Genotypen (n) & $\begin{array}{l}\text { D7 (53) } \\
\text { C2 (47) }\end{array}$ & D7 (157) & D7 (180) & $\begin{array}{l}\text { C2 (38) } \\
\text { D7 (33) }\end{array}$ & D6 (2) & D4 (25) & D6 (166) & D8 (56) & D5 (150) & D4 (124) & D4 (138) & \\
\hline $\begin{array}{l}\text { Zahl der AGM-Fälle } \\
\text { mit MV-Genotypisierung }\end{array}$ & 109 & 158 & 119 & 8 & 0 & 8 & 65 & 4 & 3 & 5 & 1 & 480 \\
\hline Häufigste MV-Genotypen der AGM-Fälle (n) & $\begin{array}{l}\text { C2 (47) } \\
\text { D7 (42) }\end{array}$ & D7 (142) & D7 (116) & $\begin{array}{l}\text { D7 (4) } \\
\text { C2 (3) }\end{array}$ & - & D4 (5) & D6 (60) & D4 (3) & D5 (3) & D4 (5) & B3 (1) & \\
\hline
\end{tabular}

D7 ( $\mathrm{n}=42)$, D6 $(\mathrm{n}=16), \mathrm{B} 3(\mathrm{n}=2)$ und D4 $(\mathrm{n}=1)$ auftraten, zeigte sich dieser MV-C2 in den folgenden 3 Jahren nur noch selten und wurde nach 2003 nicht mehr gefunden. Von 2001 bis 2003 wurde der MV-Genotyp D7 am häufigsten nachgewiesen, im Jahr 2005 war der Genotyp D4 vorherrschend und im Jahr 2006 Genotyp D6. Bis zum Jahr 2002 kam die Mehrzahl der sequenzierten Proben aus dem Masern-Sentinel. Der Anteil der AGMProben an allen im NRZ sequenzierten Proben ging seit dem Jahr 2003 zurück, die häufigsten Genotypen waren bis 2006 auch in den AGM-Proben vertreten. Von 2007 bis 2010 wurden nur noch 13 AGMFälle im NRZ MMR sequenziert im Vergleich zu 602 Fällen, die nicht aus dem AGM-Sentinel kamen. Insgesamt wurden im Untersuchungszeitraum in 21 Fällen (darunter 4 aus dem AGM-Sentinel) der MV-Genotyp A nachgewiesen, dem exklusiv Impfviren angehören und dessen Nachweis für Impfmasern spricht.

\section{Diskussion}

Das Sentinel der AGM war das erste bundesweite Surveillancesystem für Masern in Deutschland und lieferte zügig und zeitnah epidemiologische Daten. Mit den Sentinel-Daten wurde zum ersten Mal belegt, dass Masern in Deutschland trotz empfohlener 2-maliger Impfung noch ein relevantes Problem darstellen [4, 14, 17].
Das Sentinel gab Auskunft über die Alters- und regionale Verteilung der Masern in Deutschland, als die Meldepflicht noch nicht etabliert war. Mit den SentinelDaten wurde die Notwendigkeit des RKIAktionsplanes zur Masern- und Rötelneliminierung in Deutschland [8] eindrücklich bestätigt.

Die Sentinel-Ergebnisse belegten, dass die Mehrzahl der Masernfälle ungeimpft und der Hauptgrund hierfür eine ablehnende Haltung gegenüber der Masernimpfung war. Bei einer Komplikationsrate von 15\% zeigte sich außerdem, dass die Masern keine harmlose Krankheit sind. Das waren wichtige Erkenntnisse, die die Forderung nach zusätzlichen Impfaktionen und verbesserter Aufklärung von Ärzten und Eltern über die Vorteile der Masernimpfung unterstützen.

Die besondere Stärke des Sentinel-Systems bestand in der laborgestützten Surveillance, insbesondere durch die molekulargenetische Diagnostik am NRZ MMR. Hier wurde die Mehrzahl der SentinelProben untersucht, und es konnten Daten über die Verbreitung von MV-Genotypen gewonnen werden. Erst als der Anteil der AGM-Fälle an allen im NRZ MMR sequenzierten Masernfällen deutlich zurückging, konnte das Sentinel die Verteilung der MV-Genotypen in Deutschland nicht mehr valide beschreiben.

Die Sentinel-Praxen gehörten zu den niedergelassenen Ärzten in Deutschland, die systematisch Laboruntersuchungen bei Patienten mit Masernverdacht und insbesondere bei geimpften Patienten anforderten. Das Sentinel trug damit maßgeblich dazu bei, dass Laboruntersuchungen immer mehr zum diagnostischen Standard für die Überprüfung von klinischen Masern-Verdachtsfällen durch die niedergelassenen Ärzte wurden.

Aufgrund der Laboruntersuchungen bot das Sentinel eine verlässliche Datengrundlage, um den Anteil der bestätigten bzw. ausgeschlossenen und damit aus den Meldungen zu löschenden Masernfälle an allen Masernverdachtsfällen abzubilden. Die hohe Bestätigungsrate bei ungeimpften im Vergleich zur hohen Ausschlussrate bei geimpften Patienten mit Masernverdacht sprach außerdem für eine hohe Wirksamkeit der Masernimpfung.

Die unterschiedlichen Bestätigungsraten im Sentinel über die Zeit zeigten an, dass der positive prädiktive Wert (PPV) der klinischen Maserndiagnose von der Prävalenz der Erkrankung abhängt: Der PPV war höher in Jahren mit Masernausbrüchen oder endemischer Masernzirkulation und niedrig, wenn Masern nur sporadisch auftraten.

Vor dem Hintergrund dieser Ergebnisse sind Laboruntersuchungen von klinischen Masernverdachtsfällen unverzichtbar für die Masern-Surveillance in Deutschland [17]. In einer Untergruppe von gemeldeten Masernfällen aus dem 
Tab. 4 Zusammenfassende Übersicht über die Stärken und Limitationen von Meldepflicht und Sentinel-Erhebung der Masern in Deutschland

\begin{tabular}{|c|c|c|}
\hline & Meldepflicht (IfSG) & Sentinel (AGM) \\
\hline Grad der Erfassung/Bevölkerungsbezug & $\begin{array}{l}\text { Totalerfassung angestrebt (cave: Untererfassung durch } \\
\text { mangelnde Wahrnehmung der Meldepflicht) } \\
\text { Inzidenzberechnung (einschließlich altersspezifisch } \\
\text { und regional), da Bevölkerungsbezug gegeben }\end{array}$ & $\begin{array}{l}\text { Stichprobenerhebung (cave: Repräsentativität } \\
\text { der Stichprobe) } \\
\text { Bevölkerungsbezug schwierig, Berechnung } \\
\text { zeitlicher und regionaler Trends bei endemischer } \\
\text { Verbreitung }\end{array}$ \\
\hline Sensitivität/Spezifität der Fallerfassung & $\begin{array}{l}\text { Höhere Sensitivität: } \\
\text { - Erfassung sporadischer Fälle mit Masernverdacht } \\
\text { - Auch bei kleinen Fallzahlen }\end{array}$ & $\begin{array}{l}\text { Höhere Spezifität: } \\
\text { - Systematische Abklärung von Verdachtsfällen }\end{array}$ \\
\hline $\begin{array}{l}\text { Laboruntersuchung zur Bestätigung } \\
\text { der klinischen Diagnose }\end{array}$ & $\begin{array}{l}\text { Zunehmend etabliert, insbesondere durch Aktivitäten } \\
\text { der Gesundheitsämter }\end{array}$ & $\begin{array}{l}\text { Aktiv und systematisch angeboten; von Primär- } \\
\text { versorgern (Ärzten) wahrgenommen }\end{array}$ \\
\hline $\begin{array}{l}\text { Untersuchungsrate (Anteil Untersuchter } \\
\text { an allen Verdachtsfällen) }\end{array}$ & Nicht darstellbar & Berechnung möglich \\
\hline $\begin{array}{l}\text { Bestätigungsrate (Anteil bestätigter } \\
\text { Masernfälle an allen laboruntersuchten } \\
\text { Verdachtsfällen) }\end{array}$ & Nicht darstellbar & Berechnung möglich \\
\hline $\begin{array}{l}\text { Klinische Details (z. B. Erkrankungsdatum, } \\
\text { Komplikationen, Hospitalisierung) }\end{array}$ & Unvollständig & Systematisch erfasst \\
\hline $\begin{array}{l}\text { Präventionsrelevante Informationen } \\
\text { (z. B. Erhebung des Impfstatus mit genauen } \\
\text { Daten, Gründe für Nichtimpfen) }\end{array}$ & $\begin{array}{l}\text { Nachfragen der Gesundheitsämter notwendig; } \\
\text { z.T. unvollständig, nicht detailliert }\end{array}$ & Systematisch und detailliert erfasst \\
\hline $\begin{array}{l}\text { Weitere epidemiologisch relevante } \\
\text { Informationen }\end{array}$ & Ausbruchserfassung & Angabe der wahrscheinlichen Infektionsquelle \\
\hline
\end{tabular}

Sentinel wurden in einem Modell Einflussfaktoren auf die Wahrscheinlichkeit für einen positiven Laborbefund getestet. Sensitivität und Spezifität des Modells waren ausreichend bezogen auf die tatsächlich laboruntersuchten Fälle, jedoch ließ sich der Anteil wahrer Masernfälle unter den nicht getesteten nicht mit ausreichender Zuverlässigkeit vorhersagen [13].

Seit 2001 bestand das Masern-Sentinel parallel zur Meldepflicht nach IfSG. Die Stärken und Limitationen der beiden Erhebungssysteme sind in der $\bullet$ Tab. 4 zusammengefasst und werden im Folgenden beispielhaft anhand der oben beschriebenen Ergebnisse dargestellt.

Beide Surveillancesysteme bildeten den generellen Trend der zurückgehenden Masernerkrankungen im Untersuchungszeitraum ab $[7,11]$. Die regionale und die Altersverteilung der Masernfälle in beiden Systemen wichen jedoch bald deutlich voneinander ab. So bildete das Sentinel nicht die Verschiebung der Altersverteilung der Fälle von Kleinund Schulkindern zu Jugendlichen und jungen Erwachsenen ab, die sich in den IfSG-Meldedaten seit 2006 zeigte [7, 15]; es sah vielmehr über den gesamten Zeitraum die Mehrheit der Masern-Fälle bei Kindern unter 10 Jahren. Der Grund hier- für ist wahrscheinlich die Zusammensetzung der Sentinel-Praxen mit dem hohen Anteil an pädiatrischen Praxen.

Eine Auswertung der Meldedaten nach IfSG zeigte, dass die Mehrheit der Masernfälle seit 2005 im Rahmen zeitlich und räumlich beschränkter Ausbrüche auftrat $[7,15,16]$. Obwohl die Verteilung der Sentinel-Praxen über die Bundesländer als repräsentativ für die Verteilung der Pädiater und Hausärzte nach Bundesländern angesehen werden konnte, wurden diese Ausbrüche nur dann vom Sentinel erfasst, wenn im betroffenen Landkreis oder der Gemeinde eine Sentinel-Praxis vertreten war. Aus demselben Grund wurden auch sporadisch auftretende Fälle nicht durch das Sentinel erfasst. Mit dem generellen Rückgang der Masernzirkulation in Deutschland verlor das Sentinel an Sensitivität. Augenfällig wurde die relative Anfälligkeit der Sentinel-Surveillance auch in einem Ausreißer der Fallzahlen im Jahr 2002, als 687 Fälle aus einem Masernausbruchs von 2 der 160 Sentinel-Praxen in Bayern gemeldet wurden, was $87 \%$ der Fälle aus Bayern und zwei Drittel aller Sentinel-Fälle des Jahres 2002 ausmachte [2, 9].

Obwohl das Sentinel nicht für die Bestimmung von Inzidenzen geeignet war, ermöglichte es, Trends der Erkrankungs- häufigkeit zu bestimmen und zusätzliche Informationen zur Meldepflicht beizubringen, insbesondere hinsichtlich des Impfstatus, von Masernkomplikationen und der Bestätigungsrate. Damit ergänzte das Sentinel die epidemiologischen Daten der Meldepflicht (• Tab. 4). Darüber hinaus trug der Parallelbetrieb beider Systeme zur Verbesserung der Masernsurveillance bei und ergab wertvolle Erkenntnisse zur Überarbeitung von Präventionsstrategien.

Im Untersuchungszeitraum verbesserte sich die Qualität der IfSG-Meldedaten zunehmend. Masernausbrüche wurden aufgrund der Meldepflicht durch den öffentlichen Gesundheitsdienst aufgedeckt und untersucht, und damit stieg auch die Zahl von Laboruntersuchungen an $[15,16$, 18]. Daneben wurden weitere Datenquellen, wie z. B. Abrechnungsdaten der Kassenärztlichen Vereinigungen, als mögliche Sekundärquelle für die Bestimmung der Zahl der Masernfälle herangezogen [6].

Mit der sinkenden Fallzahl ging die Relevanz des Sentinels für die Masernsurveillance zurück, und das AGM-Sentinel wurde im April 2011 eingestellt.

Die Sentinel-Surveillance mit einer selbst selektierten Stichprobe von Melde- 
praxen erwies sich als effektives Mittel für die Surveillance von häufig auftretenden (endemischen) Krankheiten. Sie erlaubte das Beschreiben von Trends und lieferte zusätzliche fallbezogene Informationen aus dem Routinebetrieb von niedergelassenen Arztpraxen. Allerdings war ohne Bestimmung eines Nenners (Bevölkerung unter Beobachtung) keine Inzidenzberechnung möglich. Wenn der Bevölkerungsbezug bekannt und die Teilnehmerdichte groß genug für die Detektion seltener Fälle ist, können Sentinels eine Meldepflicht ersetzen. Ein Beispiel hierfür wäre eine Beobachtungsregion, in der alle praktizierenden Ärzte als Melder einbezogen sind und die repräsentativ sowohl in der Bevölkerungsstruktur und in dem Sinne ist, das hier Fälle mit der gleichen Wahrscheinlichkeit wie in jeder anderen Region zu erwarten sind.

Repräsentativität ist eine wesentliche Voraussetzung für die Sentinel-Surveillance. Die Zusammensetzung der Sentinel-Praxen sollte es ermöglichen, Fälle aller Alters- und Bevölkerungsgruppen zuverlässig zu finden. Die Selbst-Selektion von Arztpraxen birgt die Gefahr dass die stärker motivierten oder dem Impfgedanken verpflichtete Praxen teilnehmen. Dem könnte durch geeignete Anreize für die Teilnehmer entgegengewirkt und damit die generelle Motivation zur Teilnahme erhöht werden.

\section{Korrespondenzadresse}

\section{Dr. A. Siedler}

Abteilung für Infektionsepidemiologie, Fachgebiet Impfprävention,

Robert Koch-Institut

DGZ-Ring 1, 13086 Berlin

siedlera@rki.de

Danksagung. Wir danken allen Arztpraxen, die in der Arbeitsgemeinschaft Masern beteiligt waren, für ihre jahrelange aktive Meldebereitschaft

Interessenkonflikt. A. Siedler und A. Mankertz geben an, dass kein Interessenkonflikt besteht. Für A. Grüber vom Deutschen Grünen Kreuz (DGK) wird auf folgende Beziehung hingewiesen: Im Rahmen der Arbeitsgemeinschaft Masern wurde die Arbeit des DGK von den Impfstoffherstellern Sanofi Pasteur MSD GmbH und GlaxoSmithKline GmbH \& Co. KG gemeinsam finanziert.

Dieser Beitrag beinhaltet keine Studien an Menschen oder Tieren.

\section{Literatur}

1. o $A$ (2001) Gesetz zur Verhütung und Bekämpfung von Infektionskrankheiten beim Menschen (Infektionsschutzgesetz - IfSG). Bundesgesetzblatt

2. Arenz S, Kalies H, Ludwig M et al (2003) Der Masernausbruch in Coburg - Was lässt sich daraus lernen? Dtsch Ärztebl 100:5

3. Faensen D, Claus H, Benzler J et al (2006) SurvNet@ RKI - a multistate electronic reporting system for communicable diseases. Euro Surveill 11:4

4. Hellenbrand W, Siedler A, Tischer A et al (2003) Progress toward measles elimination in Germany. J Infect Dis 187:9

5. Mankertz A, Mulders MN, Shulga S et al (2011) Molecular genotyping and epidemiology of measles virus transmission in the World Health Organization European Region, 2007-2009. J Infect Dis 204:8

6. Mette A, Reuss AM, Feig M et al (2011) Under-reporting of measles: an evaluation based on data from North Rhine-Westphalia. Dtsch Arztebl Int 108:6

7. Robert Koch-Institut (2011) Infektionsepidemiologisches Jahrbuch 2010. 6.33 Masern. Robert KochInsitut, S6

8. Robert Koch-Institut (1999) Interventionsprogramm Masern, Mumps, Röteln - Konzept für ein nationales Programm zur Eliminierung der Masern in der Bundesrepublik Deutschland. http://www. rki.de/DE/Content/Infekt//mpfen/Praevention/intervt.html

9. Robert Koch-Institut (2002) Sentinel-Surveillance der Arbeitsgemeinschaft Masern (AGM). Ergebnisse bis zum Ende des 1. Halbjahres 2002. Epid Bulletin 32:5

10. Robert Koch-Institut (o J) SurvStat@RKI: Abfrage der Meldedaten nach Infektionsschutzgesetz (IfSG) über das Web. http://www.rki.de/DE/Content/Infekt/SurvStat/survstat_node.html

11. Robert Koch-Institut (2004) Zur Situation bei wichtigen Infektionskrankheiten: Erkrankungen an Masern, Mumps und Röteln (MMR). Epid Bulletin 35:5

12. Santibanez S, Tischer A, Heider A et al (2002) Rapid replacement of endemic measles virus genotypes. J Gen Virol 83:10

13. Schlaud M, Siedler A, Tischer A et al (2004) Estimating the validity of measles notifications by a prediction model. Biom J 46:1

14. Siedler A, Hermann M, Schmitt HJ, Kries R von (2002) Consequences of delayed measles vaccination in Germany. Pediatr Infect Dis J 21:5

15. Siedler A, Mankertz A, Feil F et al (2011) Closer to the goal: efforts in measles elimination in Germany 2010. J Infect Dis 204:8

16. Siedler A, Tischer A, Mankertz A, Santibanez $S$ (2006) Two outbreaks of measles in Germany 2005. Euro Surveill 11:5

17. Tischer A, Santibanez S, Siedler A et al (2004) Laboratory investigations are indispensable to monitor the progress of measles elimination - results of the German Measles Sentinel 1999-2003. J Clin Virol 31:14

18. Wichmann O, Siedler A, Sagebiel D et al (2009) Further efforts needed to achieve measles elimination in Germany: results of an outbreak investigation. Bull World Health Organ 87:8
Ethische Entscheidungswege in der Biomedizin

Jahrestagung 2013 der Akademie der Ethik in der Medizin

In der modernen Biomedizin bestehen zahlreiche ethische Konflikte und Dilemmata. Dies liegt an der sachlichen Komplexität des Handlungsbereichs sowie an der Vielschichtigkeit moralischer Überzeugungssysteme in pluralen Gesellschaften. Obwohl es nicht immer gelingt, eine allgemein akzeptierte Lösung zu finden, sind Entscheidungen zu treffen. Hierzu müssen konfligierende ethische Normen, Werte, Ideale und Interessen abgewogen werden. Wie diese Abwägungen zustande kommen, ist in vielen Fällen nicht transparent. Etablierte Methoden der ethischen Abwägung derartiger Konfliktsituationen fehlen bislang.

Bei vielen konkreten medizinethischen Entscheidungen setzt man daher auf prozedurale Lösungsansätze. Nicht zufällig haben ethische Kommissionen und Komitees in der Biomedizin (und darüber hinaus) Konjunktur. Aber auch hier müssen die Akteure letztlich pragmatische Entscheidungen treffen.

Die Tagung möchte Licht in die Abwägungs- und Entscheidungsprozesse bei medizinethischen Fragen werfen: Welche normativen Methoden der Abwägung und Entscheidungsfindung stehen zur Verfügung? Wie kommen Abwägungen in der Praxis zustande? Wie sind ethische Theorien und praktische Entscheidungen verbunden? Welche Rolle spielen die moralische Urteilskraft bzw. moralische Intuitionen in Theorie und Praxis? Wie sollten Abwägungsprozesse formal organisiert werden? Wie laufen Entscheidungen in ethischen Komitees und Kommissionen $a b$ ? Nach welchen Kriterien werden ihre Mitglieder ausgewählt? Wie verhalten sich ethische Abwägungen zu rechtlichen Abwägungen, politischen Kompromissen und pragmatischen Entscheidungen im Einzelfall?

Datum und Ort:

10.-12. Oktober 2013, München

Information: Geschäftsstelle der Akademie für Ethik in der Medizin e. V.

Tel.: +49 (0)551 39-9680,

E-Mail: info@aem-online.de 